\title{
THE GEOMETRY OF THE MODULI SPACE OF RIEMANN SURFACES
}

\author{
BY SCOTT A. WOLPERT ${ }^{1}$
}

We wish to describe how the hyperbolic geometry of a Riemann surface of genus $g, g \geq 2$, leads to a symplectic geometry on $T_{g}$, the genus $g$ Teichmüller space, and $\bar{M}_{g}$, the moduli space of genus $g$ stable curves. The symplectic structure has three elements: the Weil-Petersson Kähler form, the FenchelNielsen vector fields $t_{*}$, and the geodesic length functions $l_{*}$. Weil introduced a Kähler metric for $T_{g}$ based on the Petersson product for automorphic forms; the metric is invariant under the covering of $T_{g}$ onto $\mathcal{M}_{g}$, the classical moduli space of Riemann surfaces [1].

To a geodesic $\alpha$ on a marked Riemann surface $\hat{R}$ with hyperbolic metric is associated the Fenchel-Nielsen vector field $t_{\alpha}$ on $T_{g} ; t_{\alpha}$ is the infinitesimal generator of the flow given by the Fenchel-Nielsen (fractional twist) deformation for $\alpha$ [7]. The infinitesimal generators of Thurston's earthquake flows form the completion (in the compact-open topology) of the Fenchel-Nielsen vector fields [6]. A basic invariant of the geodesic $\alpha$ on a marked surface $\hat{R}$ is its length $l_{\alpha}(\hat{R})$; the exterior derivative $d l_{\alpha}$ is a 1 -form on $T_{g}$. We have the following formulas for the Weil-Petersson Kähler form $\omega$ [8].

THEOREM 1.

$$
\begin{aligned}
\omega\left(t_{\alpha},\right)= & -d l_{\alpha}, \\
\omega\left(t_{\alpha}, t_{\beta}\right) & =\sum_{p \in \alpha^{*} \beta} \cos \theta_{p}, \\
t_{\alpha} t_{\beta} l_{\gamma}= & \sum_{(p, q) \in \alpha^{*} \gamma \times \beta^{*} \gamma} \frac{e^{l_{1}}+e^{l_{2}}}{2\left(e^{l_{\gamma}}-1\right)} \sin \theta_{p} \sin \theta_{q} \\
& -\sum_{(r, s) \in \alpha^{\#} \beta \times \beta^{*} \gamma} \frac{e^{m_{1}}+e^{m_{2}}}{2\left(e^{l_{\beta}}-1\right)} \sin \theta_{r} \sin \theta_{s} .
\end{aligned}
$$

An immediate consequence of (i) is that the symplectic form $\omega$ is invariant under the flow of a Fenchel-Nielsen vector field. The r.h.s. of (ii), evaluated at $\hat{R} \in T_{g}$, is the sum of the cosines of the angles at the intersections of the geodesics $\alpha$ and $\beta$ on the surface $\hat{R}$. Similarly the r.h.s. of (iii) is a sum of trigonometric invariants for pairs of intersections; $l_{1}$ and $l_{2}$ are the lengths of the segments on $\gamma$ defined by $p, q$ and, likewise, for $m_{1}$ and $m_{2}$ relative to $\beta$. Finally (i) and (ii) may be combined to show that the renormalized vector

Received by the editors May 10, 1983.

1980 Mathematics Subject Classification. Primary 30A46, $14 \mathrm{H} 15$.

${ }^{1}$ Partially supported by a grant from the National Science Foundation; Alfred P. Sloan Fellow.

(C) 1984 American Mathematical Society $0273-0979 / 84 \$ 1.00+\$ .25$ per page 
fields, $N_{\alpha}=\left(4 \sinh l_{\alpha} / 2\right) t_{\alpha}$, span a Lie algebra over the integers, which admits a purely topological characterization [8].

Fenchel-Nielsen introduced real analytic coordinates for $T_{g}$ as the parameters for the construction of a hyperbolic metric on a genus $g$ surface. The twist-length coordinates $\left(\tau_{j}, l_{j}\right)$ vary freely: $\tau_{j} \in \mathbf{R}, l_{j} \in \mathbf{R}^{+}[\mathbf{6}, 10]$. An immediate consequence is that $T_{g}$ is a cell: $T_{g} \approx\left(\mathbf{R}^{+} \times \mathbf{R}\right)^{3 g-3}$. The Kähler form $\omega$ is given simply in Fenchel-Nielsen coordinates $[\mathbf{1 0}]$.

Theorem 2. $\omega=-\sum_{j} d \tau_{j} \wedge d l_{j}$.

In particular, the data $\left(\omega, \tau_{j}, l_{j}\right)$ represent a completely integrable Hamiltonian system. Using the above formula it is readily checked that $\omega$ extends to a symplectic form on $\bar{M}_{g}$, the moduli space of stable curves (hyperbolic Riemann surfaces with nodes). Denote by $\omega^{F N}$ this extension of $\omega$ to $\bar{M}_{g}$.

Our most recent efforts have been directed towards establishing the following.

TheOREM 3. An integral multiple of $\omega / \pi^{2}$ is the Chern form of a positive line bundle over $\bar{M}_{g}$.

Let us sketch the development of this result. In [9], using formula (i) above, the integral $\int_{\mathcal{M}_{1,1}} \omega, \mathcal{M}_{1,1}$ the moduli space of once punctured tori, was calculated: the value is $\pi^{2} / 6$. Masur showed that the Weil-Petersson metric in complex coordinates is actually singular for vectors transverse to $D=\bar{M}_{g}-\mathcal{M}_{g}$, the divisor of surfaces with nodes, [5]. Nevertheless in [10] it was shown that $\omega^{\mathbf{C}}$, the extension to $\bar{M}_{g}$ of the Kähler form considered in complex coordinates, is a closed, type $(1,1)$ current and $\omega^{\mathbf{C}}$ and $\omega^{F N}$ represent the same cohomology class in $H^{2}\left(\bar{M}_{g} ; \mathbf{R}\right)$. In [11] we considered the generalization of the earlier $\pi^{2} / 6$ result: $\omega / \pi^{2} \in H^{2}\left(\bar{M}_{g} ; \mathbf{Q}\right)$. But first note that the divisor $D=\bar{M}_{g}-\mathcal{M}_{g}$ is reducible, $D=D_{0} \cup \cdots \cup D_{\lfloor g / 2\rfloor}$, where the generic surface $R$ represented in $D_{k}$ has one node separating $R$ into components of genus $k$ and genus $g-k$. Certainly the divisors $D_{k}$ define cohomology classes in $H_{6 g-8}\left(\mathcal{M}_{g}\right)$, and by Poincaré duality $\omega$ also determines a class in $H_{6 g-8}\left(\bar{M}_{g}\right)$.

Theorem 4. $\left\{\omega / \pi^{2}, D_{0}, \ldots, D_{\lfloor g / 2\rfloor}\right\}$ is a basis for $H_{6 g-8}\left(\mathcal{M}_{g} ; \mathbf{Q}\right)$.

The plan of the proof of Theorem 4 is straightforward. By the work of Harer [4] and an application of Mayer-Vietoris, one checks beforehand that $\mathrm{H}_{2}\left(\mathcal{M}_{g} ; \mathbf{Q}\right)$ has rank $2+\lfloor g / 2\rfloor$. A candidate basis is then presented for each of $\mathrm{H}_{2}\left(\overline{\mathcal{M}}_{g}\right)$ and $\mathrm{H}_{6 g-8}\left(\overline{\mathcal{M}}_{g}\right)$, and the intersection pairing is evaluated [11]. The pairing is nonsingular and so bases have been given for the homology groups $\mathrm{H}_{2}$ and $\mathrm{H}_{6 g-8}$. By similar techniques it may also be shown that the rank of $H_{2 k}\left(\bar{M}_{g}\right), k<g$, is at least

$$
\frac{1}{2}\left(\begin{array}{c}
g-1 \\
k
\end{array}\right)
$$

To complete the proof of Theorem 3 it only remains to check that $\omega$ is cohomologous in the sense of currents to a closed, positive, $(1,1)$ form. Let $\Omega$ be that multiple of $\omega / \pi^{2}$ which represents a class in $H^{2}\left(\overline{\mathcal{M}}_{g} ; \mathbf{Z}\right)$. Now the formalism of Chern classes extends to currents: $\Omega$ determines the class of a 
line bundle over $\bar{M}_{g}$. By direct potential theoretic estimation it is shown that $\Omega$ is the curvature form, again in the sense of currents, of a continuous metric in a line bundle [12]. Next it is checked that $\omega$ is bounded below by a smooth positive $(1,1)$ form; $\Omega$ is a positive current. Finally, standard techniques from the study of plurisubharmonic functions may be used to complete the argument [12].

$\bar{M}_{g}$ is a compact, complex $V$-manifold and so we may refer to Baily's version of the Kodaira Imbedding Theorem [2].

TheOREM 5. The positive line bundle associated to the Weil-Petersson Kähler form gives rise to a projective embedding: $\bar{M}_{g} \hookrightarrow \mathbf{C P}$.

\section{REFERENCES}

1. L. V. Ahlfors, Some remarks on Teichmüller's space of Riemann surfaces, Ann. of Math. (2) 74 (1961), 171-191.

2. W. L. Baily, On the imbedding of $V$-manifolds in projective space, Amer. J. Math. 79 (1957), 403-430.

3. L. Bers, Spaces of degenerating Riemann surfaces, Ann. of Math. Stud. 79 (1974), 43-55.

4. J. Harer, The second homology group of the mapping class group of an orientable surface, Invent. Math. 72 (1982), 221-239.

5. H. Masur, The extension of the Weil-Petersson metric to the boundary of Teichmüller space, Duke Math. J. 43 (1976), 623-635.

6. W. P. Thurston, The geometry and topology of 3-manifolds, notes.

7. S. A. Wolpert, The Fenchel-Nielsen deformation, Ann. of Math. (2) 115 (1982), 501-528.

8. (2) 117 (1983), 207-234.

9. - On the Kähler form of the moduli space of once punctured tori, Comment. Math. Helv. 58 (1983), 246-256.

10. , On the Weil-Petersson geometry of the moduli space of curves, Amer. J. Math. (to appear).

11. - On the homology of the moduli space of stable curves, Ann. of Math. (2) 118 (1983), 491-523.

12. __ On obtaining a positive bundle from the Weil-Petersson class, Amer. J. Math. (to appear).

13. - Chern forms and the Riemann tensor for the moduli space of curves, preprint.

Department of Mathematics, University of Maryland, College Park, MARYLAND 20742 
